\title{
Transmission of new CRF07_BC Strains with 7 amino acid deletion in Gag p6
}

Meng Zhefeng ${ }^{1}$, Hu Huiliang ${ }^{1,2}$, Qiu Chao ${ }^{1}$, Sun Jun ${ }^{1,2}$, Lu Jianxin ${ }^{2}, Z^{2}$ hang Xiaoyan ${ }^{1,3^{*}}$, Xu Jianqing ${ }^{1,3^{*}}$

\begin{abstract}
A 7 amino acid deletion in Gag p6 (P6delta7) emerged in Chinese prevalent HIV-1 strain CRF07_BC from different epidemic regions. It is important to determine whether this mutation could be transmitted and spread. In this study, HIV-1 Gag sequences from 5 different epidemic regions in China were collected to trace the transmission linkage and to analyze genetic evolution of P6delta7 strains. The sequence analysis demonstrated that P6delta7 is a CRF07_BC specific deletion, different P6delta7 strains could be originated from different parental CRF07_BC recombinants in different epidemic regions, and the transmission of P6delta7 strain has occurred in IDU populations. This is for the first time to identify the transmission linkage for P6delta7 strains and serves as a wakeup call for further monitoring in the future; In addition, P6delta7 deletion may represent an evolutionary feature which might exert influence on the fitness of CRF07_BC strain.
\end{abstract}

\section{Findings}

Several studies reported that mutations in HIV-1 Gag p6 played no role or only marginal role in the infection and the replication of HIV-1 in vitro[1-3]. However, it remains unknown whether those mutations in $\mathrm{p} 6$ could exert influences on HIV-1 during natural infection and thereby cause the transmission of those mutated strains. Recently, a new Gag p6 mutation pattern, 7 amino acid (aa) deletion in the central region of p6 domain (PIDKELY at amino acid 30-36, designated as P6 $\Delta 7$ ), emerged in CRF07_BC infected individuals in Xinjiang Uygur Autonomous Region of China and has progressively affected nearly 30\% CRF07_BC infected population [4]. In addition, P $6 \Delta 7$ deletion was also identified in CRF07_BC strains circulating in other epidemic sites in China mainland and even in Taiwan region [5-9].

Interestingly, though early cross-sectional observation by Song et al did not observe significant influences of P6 47 mutation on biological properties of CRF07_BC, prolonged longitudinal follow-up revealed that P6 67 deletion might result in the improvement of CRF07_BC fitness in vivo. First, after P6 $\Delta 7$ mutation occurs in vivo,

\footnotetext{
*Correspondence: zhang_xycn2002@yahoo.com.cn; jianqingxu2008@gmail. com

${ }^{1}$ Shanghai Public Health Clinical Center, Institutes of Biomedical Sciences, Fudan University, 2901 Caolang Road, Research Center, Jinshan District,

Shanghai, China, 201508

Full list of author information is available at the end of the article
}

the mutated strain will subsequently replace its parental strain and become the predominant strain; In contrast, the reversion from $\mathrm{P} 6 \Delta 7$ strain to non-P $6 \Delta 7$ strain has never been observed so far; Second, viral loads in P $6 \Delta 7$ CRF07_BC infected subjects will be more rapidly increased than that in non-P6 $\Delta 7$ strain infected individuals (Additional file 1, unpublished data). These data suggested that $\mathrm{P} 6 \Delta 7$ deletion may have important implications for CRF07_BC prevalence.

Since CRF07_BC strain is one of the most prevalent HIV-1 strains in China [4-9], the appearance of $P 6 \Delta 7$ CRF07_BC strains in different epidemic regions raised several important concerns. First, does P6 $\Delta 7$ represent a feature only in CRF07_BC or also in other BC recombinant forms? Second, is P6 $\Delta 7$ strain able to transmit and spread in population? To answer those questions above, we analyzed Chinese-derived Gag full-length sequences collected from all publicly accessible databases and traced the transmission linkage among CRF07_BC P6 $\Delta 7$ strains which were derived from 4 different provinces and 1 region in China. Interestingly, P6 $\Delta 7$ was proved to be a CRF07_BC specific mutation and could be originated from different CRF07_BC strains; Furthermore, the mutant strains of $\mathrm{P} 6 \Delta 7$ could be transmitted in population in epidemic regions and thereby may cause a new prevalence in the future.

98 Chinese derived HIV-1 Gag sequences from HIV database http://www.hiv.lanl.gov, including 27 CRF07_BC,

\section{Biomed Central}


33 CRF08_BC, 31 BC URFs (unique recombinant forms), 2 India- $C$ and 5 Thai- $B$, were collected and analyzed by neighbor-joining phylogenetic tree (Figure 1). Interestingly, though CRF07_BC, CRF08_BC and other BC recombinants were derived from the same parental strains (Thai$\mathrm{B}$ and India-C), P6 7 was only identified in CRF07_BC
(Figure 1). In total, 27 full-length CRF07_BC Gag sequences were available in HIV database, these sequences were derived from 4 provinces and 1 region, including 10 from Yunnan, 8 from Liaoning, 6 from Xinjiang, 2 from Guangxi provinces and 1 from Taiwan region. Among 27 published sequences, 8 (30\%) contain P6 $\Delta 7$ mutation,

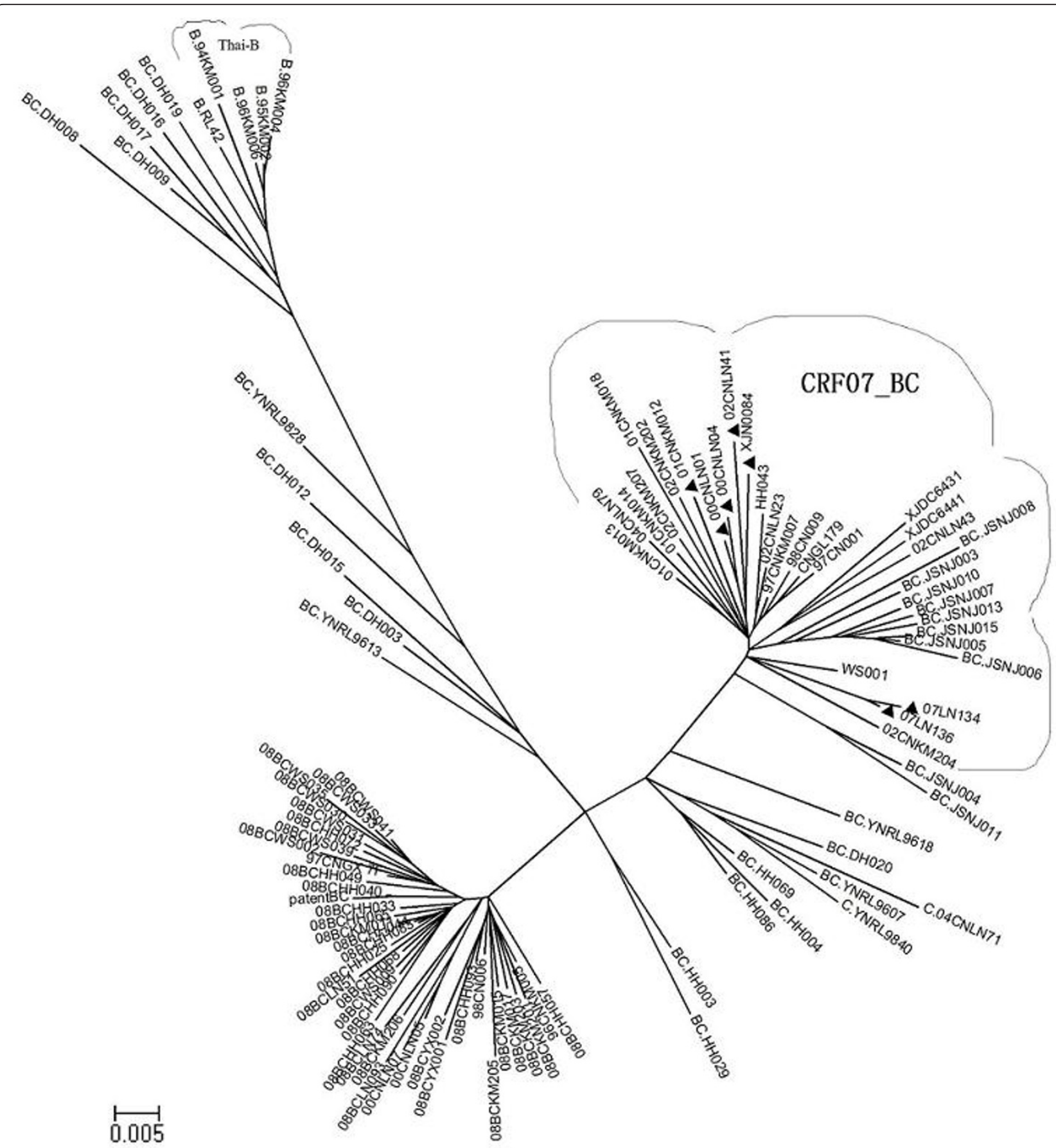

Figure 1 Phylogenetic tree constructed with 98 Chinese-derived HIV-1 BC recombinant gag sequences from HIV database. The neighbor-joining tree was constructed by Mega4.0. CRF07_BC strains forms an independent cluster and P6 7 strains were all clustered within CRF07_BC strains. $\Delta$ represents $P 6 \Delta 7$ strains. 
which is in accordance with previous report in population level that this deletion appeared in 30\% CRF07_BC recombinant infected subjects from Xinjiang province [4].

To analyze 27 CRF07_BC sequences, the general timereversible model with a proportion of invariant sites and gamma distribution $(\mathrm{GTR}+\gamma+\mathrm{R})$ was selected as the most appropriate analysis model by Modeltest software [10] and subsequently phylogenetic trees were reconstructed by using a maximum likelihood (ML) heuristic search in PAUPv4.0b10 [11]. Two P6 $\Delta 7$ sequences (07LN134 and 07LN136) were clustered in one branch with high bootstrap (Figure 2), and epidemiological

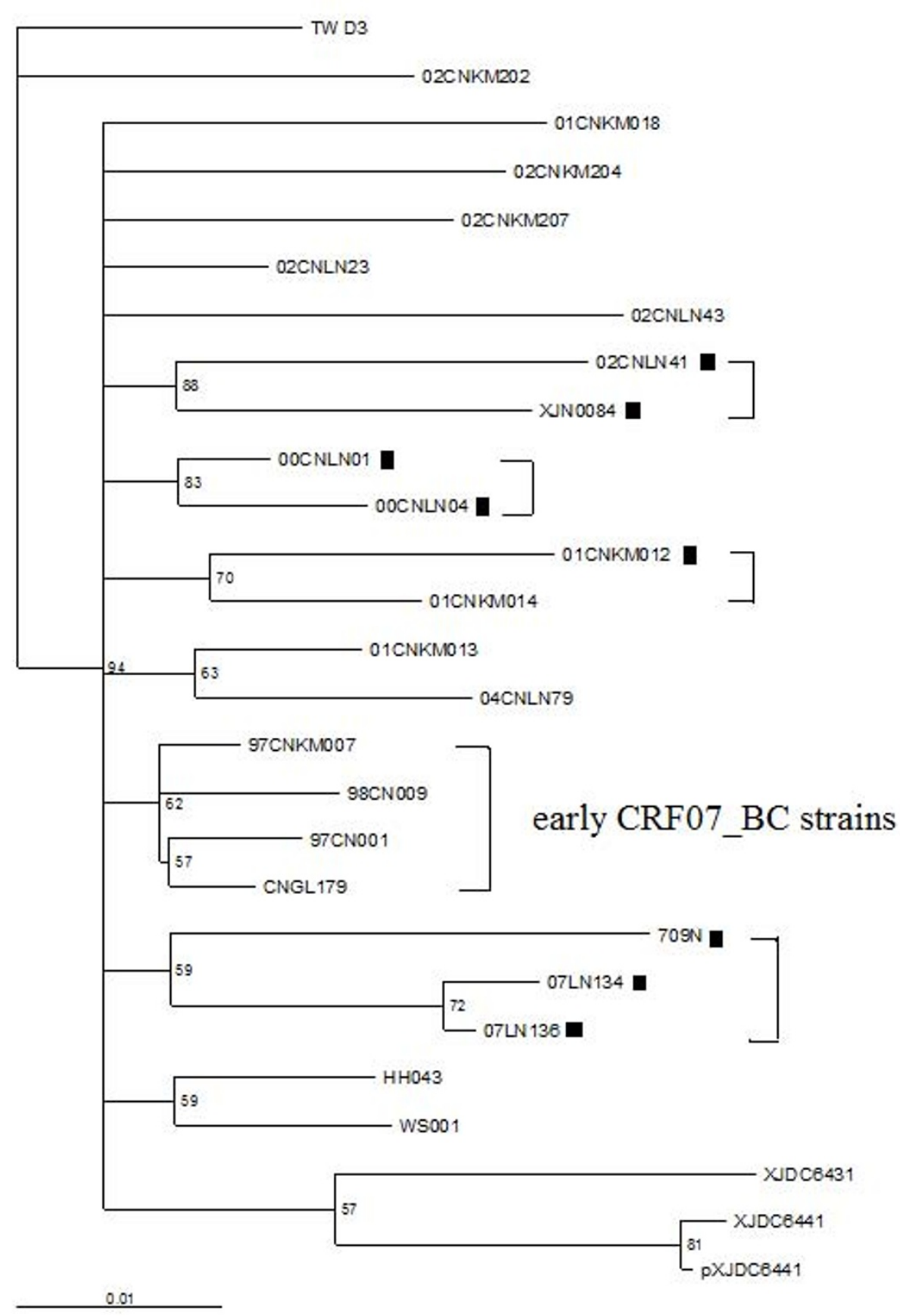

Figure 2 Maximum-likelihood tree of $\mathbf{2 7}$ CRF07_BC Gag sequences derived from HIV database. 01 CNKM012 and 01CNKM014, 00 CNLN01 and 00CNLN04, 02CNLN41 and 709, 07LN134 and 07LN136 were paired respectively by more than 70\% bootstrap probability and identified as a transmission linkage. $\mathbf{I}$ represents P6 7 CRF07_BC strains. 
study demonstrated that they were derived from an IDU couple in Liaoning province, China. Another cluster of P6 47 sequences was observed for 00CNLN01 and 00CNLN04, and these two sequences were derived from two IDUs who had shared injection needles during their intravenous drug usage. For these two clusters, both Kishino-Hasegawa test and Shimodaira-Hasegawa test $[12,13]$ showed that the transmission linkage was accepted by $\mathrm{p}$ value $>0.95$.

To test the compatibility of the reconstructed evolutionary relationship with the proposed transmission linkage, the ML trees with different tree topologies were compared by using the Kishino-Hasegawa test and the Shimodaira-Hasegawa test in Consel [12,13]. As shown in Figure 3, all tree topologies are compatible with the hypothetical transmission from $709 \mathrm{~N}$ to $07 \mathrm{LN} 134$ and 07LN136. However, in this case, the original donor may be varied in the tree depending on possible transmission. To test compatibility under this circumstance, we tested any of these topologies against ML tree. The reconstructed maximum-likelihood tree (Figure 4) showed that all P6 $\Delta 7$ strains were clustered together with early CRF07_BC strains (97CNKM007, 97CN001, CNGL179, and $98 \mathrm{CN} 009$ ), which were not rejected by the KishinoHasegawa test and the Shimodaira-Hasegawa test with $\mathrm{p}$ value in the range of $0.05 \sim 0.95$, indicating those P $6 \Delta 7$ strains could be originated from early non-deletion BC recombinant ancestor strain. However, the possibility that all P6 $\Delta 7$ strains form one cluster (Figure 5) was strongly rejected by the Kishino-Hasegawa test and the Shimodaira-Hasegawa test $(\mathrm{p}=0.013)$, suggesting those P $6 \Delta 7$ strains are not derived from the same deletion ancestor strain. Furthermore, two important observations

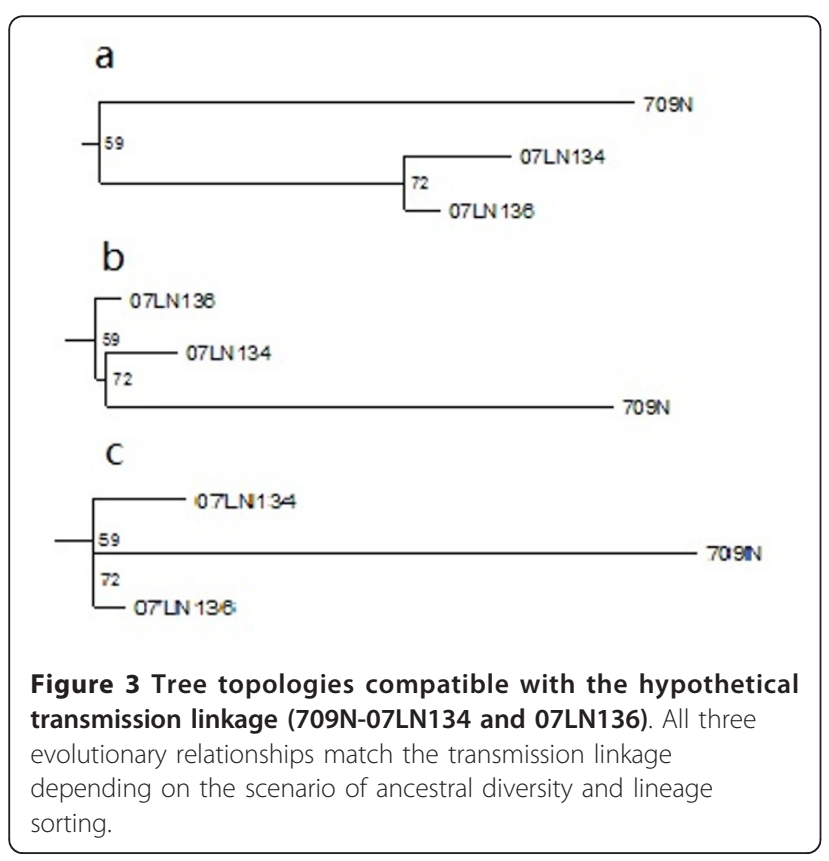

should be noticed. First, as the earliest identified CRF07_BC P6 7 isolates, 00CNLN01 and 00CNLN04 had no obvious transmission linkage with other P $6 \Delta 7$ isolates even in the same epidemic region (Liaoning province); Second, Yunnan province was considered as BC recombinant originated region $[6,8,9]$, however, $\mathrm{P} 6 \Delta 7$ isolate (01CNKM012) from Kunming city in Yunnan province was not clustered with other P $6 \Delta 7$ isolates, instead, this strain clustered with non-P $6 \Delta 7$ Kunming isolate 02CNKM014. Overall, these data further supported that different P $6 \Delta 7$ strains could be independently originated and the transmission of P $6 \Delta 7$ strains only occurred among IDUs who are closely related and thereby could be defined at the very early phase.

To further confirm the transmission linkage, Bayesian phylogenetic inference was also performed by employing Markov chain Monte Carlo (MCMC) sampling approach in $\mathrm{GTR}+\gamma+\mathrm{R}$ model, as implemented in MrBayes 3.1 $[11,14]$. The MCMC search was run for $10^{7}$ generations with trees sampled every 1000th generation. Burn-in was set at $50 \%$ and a posterior consensus tree was generated from 25,000 trees sampled. The posterior probability of nodes on the consensus tree was used as phylogenetic support for clusters. Based on previously reports $[11,14]$, significant linkages were considered as those having bootstrap values $>90 \%$ and genetic distances $<0.03$ nt substitutions per site for gag sequences. As expected, the Maximum-likelihood tree constructed by Bayes method also confirmed the transmission linkage of 07LN134 and 07LN136, 00CNLN01 and 00CNLN04, 01CNKM012 and 01CNKM014 (Figure 6). Interestingly, although the pairs were supported by high bootstrap for sequence clusters of 02CNLN41 and XJN0084, 709N and 07LN134/07LN136, the transmission linkage was not supported by both the KishinoHasegawa test and the Shimodaira-Hasegawa test with $\mathrm{p}$ value below 0.95 , and those sequences were actually derived from IDUs who reside in different provinces by thousands miles apart. These data indicated that approaches employed here to test the transmission linkage are reliable and CRF07_BC P6 67 strains could be transmitted among IDUs.

The same analysis was performed for additional 43 CRF07_BC gag sequences which were collected from IDU subjects in Urumqi city in Xinjiang Uygur Autonomous Region, China, as described previously [4]. Maximum-likelihood tree was constructed by MrBayes 3.1 (Figure 7). Among 43 sequences, 10 sequences were identified containing P $6 \Delta 7$ mutations. Similar to the results from the analysis above, the transmission linkages between P6 $\Delta 7$ isolates (XJN0301 and CBJB309), or between $\mathrm{P} 6 \Delta 7$ isolates and non-P $6 \Delta 7$ isolates (CBJB069 and XJN017) were supported by both Kishino-Hasegawa test and Shimodaira-Hasegawa test (p 


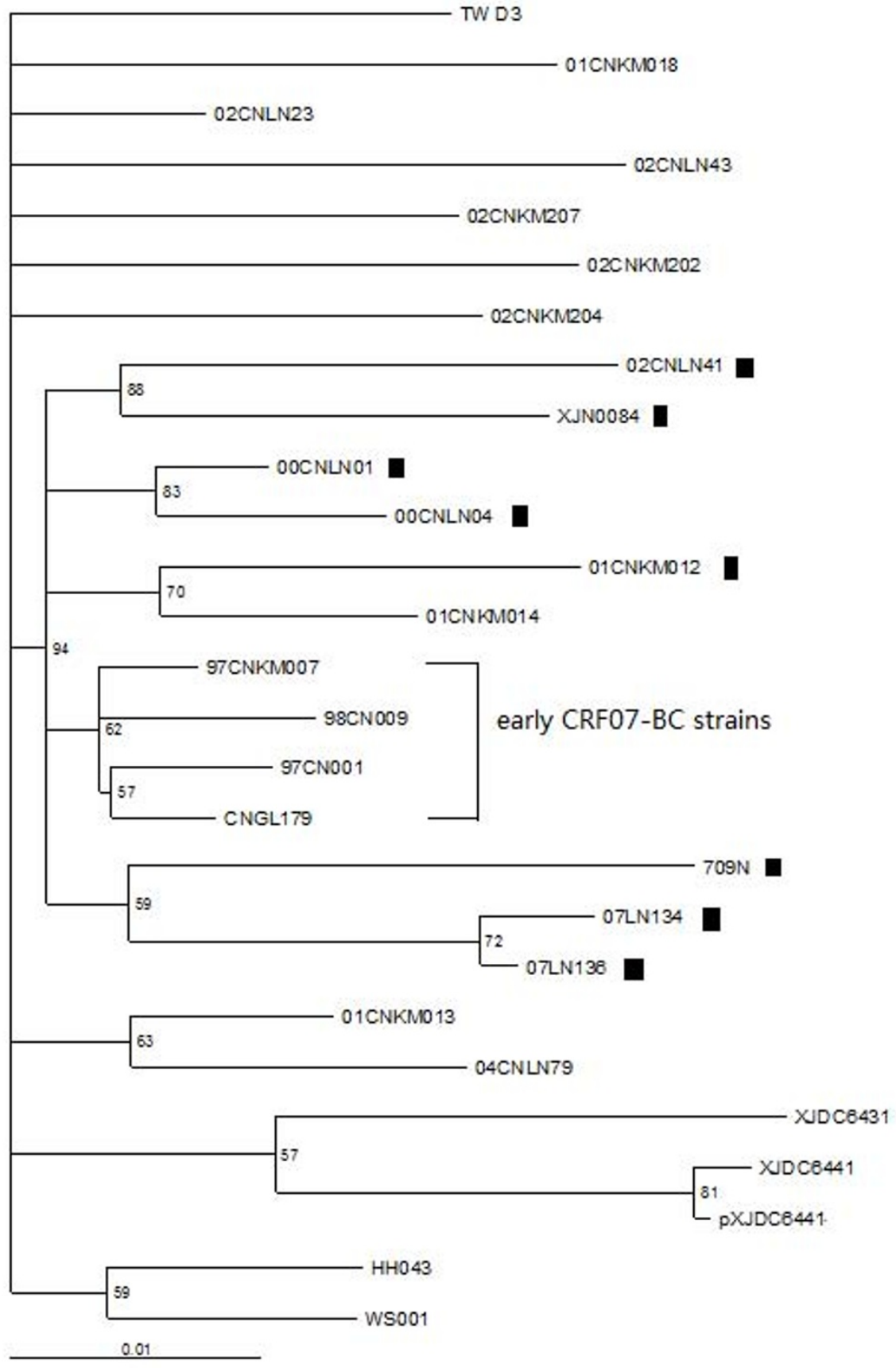

Figure 4 Reconstructed Maximum-likelihood tree against Figure 2. P6 $\triangle 7$ CRF07_BC strains clustered with early CRF07_BC isolate sequences

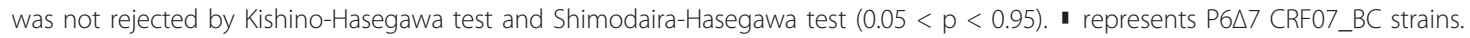

$>0.95)$. These data confirmed the observations above that P $6 \Delta 7$ strains could be originated independently and the transmission of $\mathrm{P} 6 \Delta 7$ strains did occur.

It remains controversial whether mutations in $\mathrm{p} 6$ could exert influence on the infection and the replication of HIV-1. Pikora CA et al and Bleiber G et al showed that deletion up to 18 aa (S14-I31) in p6 only had minor effects on the infectivity of HIV in vitro
$[2,3]$; In contrast, Lazert $C$ et al observed that 4 aa deletion in the central region of p6 $(\Delta 25 \mathrm{SQKQ} 28)$ increased its association with ALIX which serves as a chaperone protein to facilitate the viral assembling and budding process [15]. Different from the artificial deletions in p6 as described above, P $6 \Delta 7$ deletion in CRF07_BC strains is naturally occurred as a unique mutation pattern in a specific subtype, which suggested 


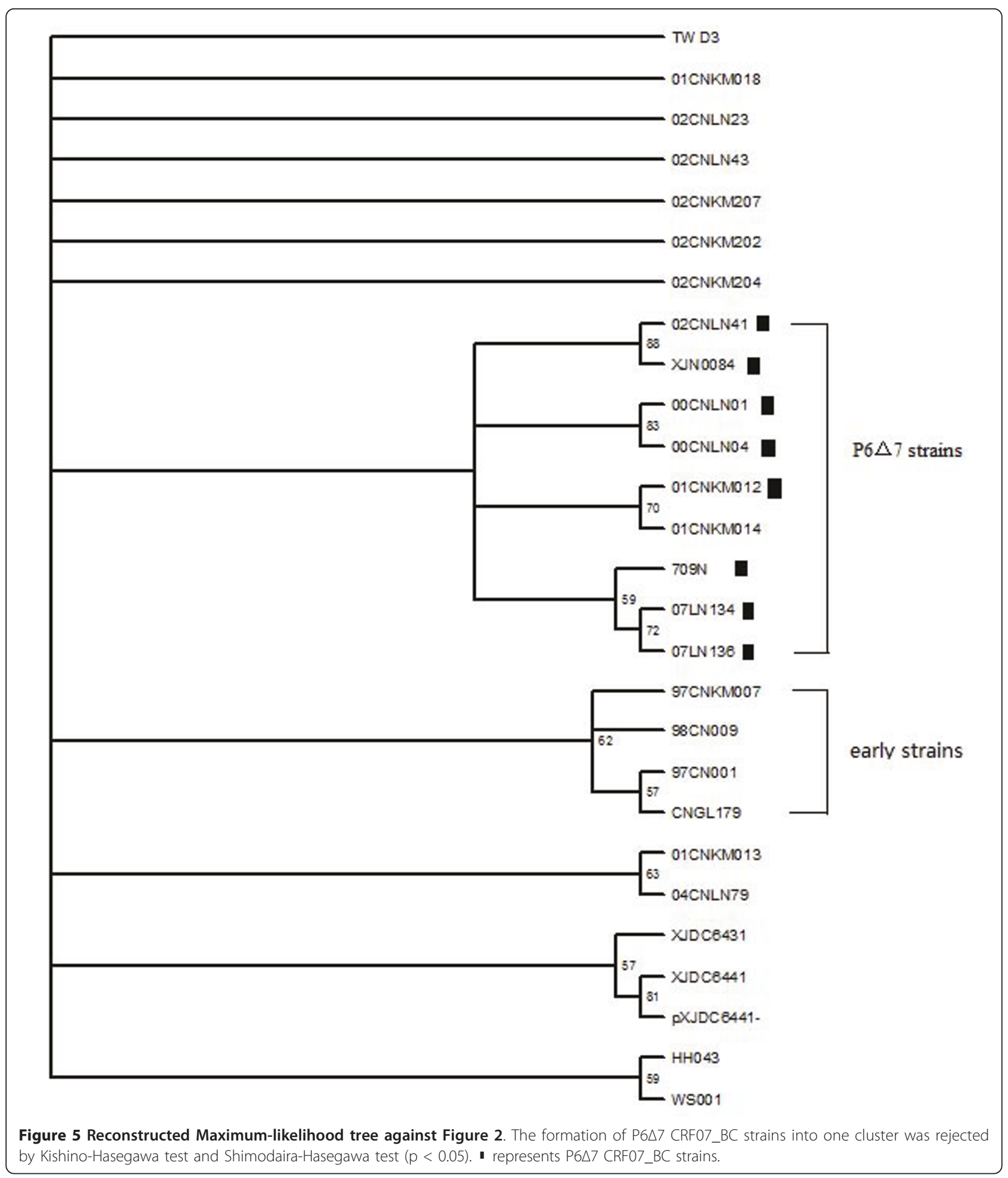

that this mutation may have important implication for CRF07_BC; Indeed, our longitudinal follow-up observed that $\mathrm{P} 6 \Delta 7$ deletion resulted in the rapid increase in viral loads (Additional file1). In addition, this deletion was not observed in CRF08_BC. As p6
Gag in CRF07_BC is derived from B clade whereas p6 Gag in CRF08_BC is from C clade, and the backbone of $\mathrm{CRF}$ _BC is derived from $\mathrm{C}$ clade, $\mathrm{P} 6 \Delta 7$ deletion may represent a new adaption of $\mathrm{B}$ clade derived $\mathrm{p} 6$ Gag to $\mathrm{C}$ clade derived backbone. 


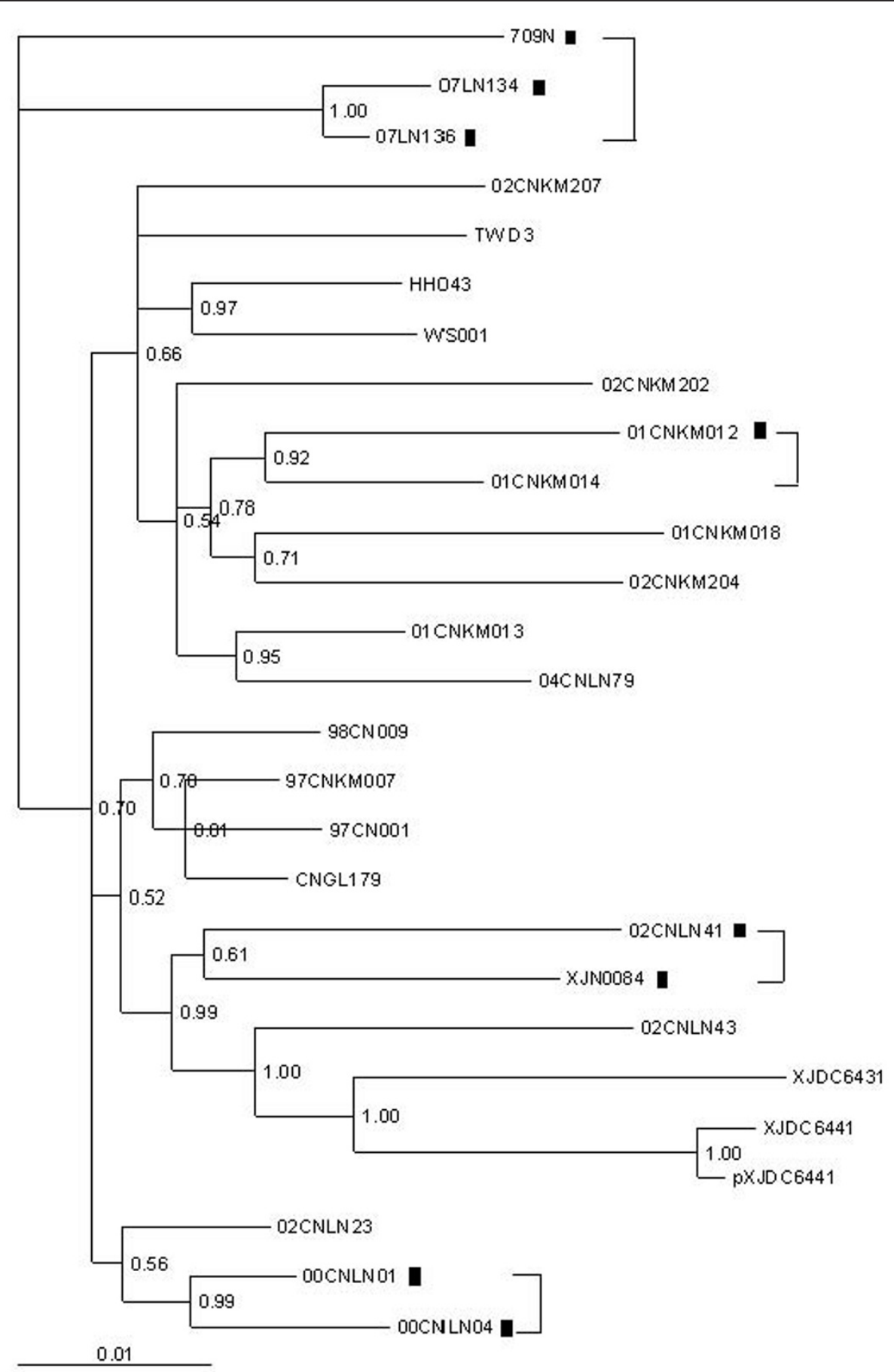

Figure 6 Maximum-likelihood tree of 27 CRF07_BC Gag sequences constructed by Bayes 3.1. 01CNKM012 and 01CNKM014, 00 CNLN01 and 00CNLN04, 02CNLN41 and 709, 07LN134 and 07LN136 are paired respectively by more than 90\% bootstrap probability. I represent represents P6 $\triangle 7$ CRF07_BC strains.

CRF07_BC is the most prevalent Chinese strains and accounts for nearly half of HIV-1 infection across the nation [4-9], suggesting that this recombinant has been highly adapted in Chinese population and any mutations in this strain needs to be closely monitored. P $6 \Delta 7$ mutation was observed in a fraction of CRF07_BC infected subjects [4], the next important question for public health is whether this mutation could be transmitted and spread. Our sequence analysis demonstrated that the transmission of P6 $\Delta 7$ strains did occur in populations. This is for the 


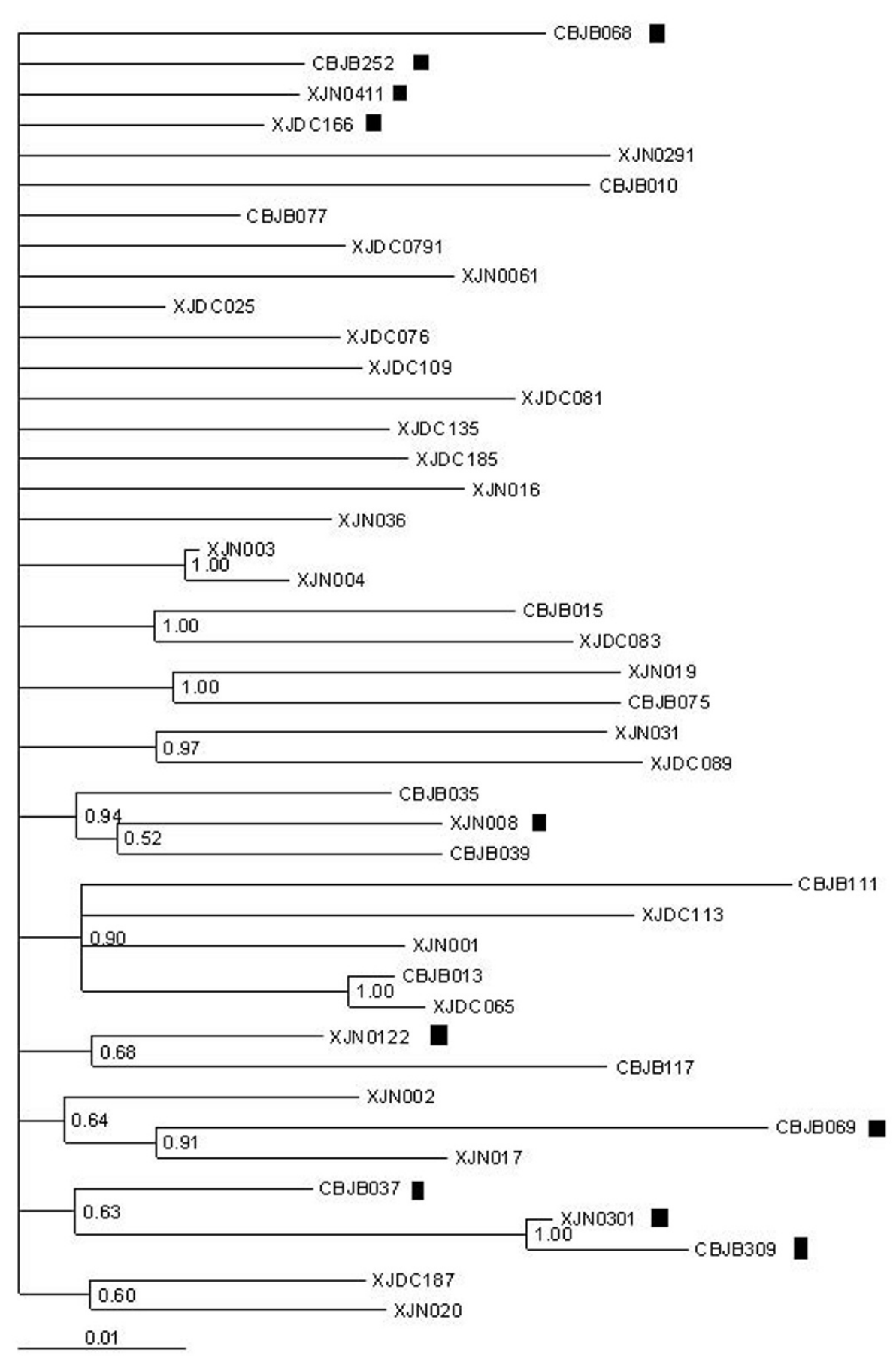

Figure 7 Maximum-likelihood tree of 43 CRF07_BC Gag sequences from Xinjiang Uygur Autonomous Region of China was constructed by Bayes3.1. XJN0301 and CBJB309, XJN017 and CBJB069 are paired respectively by more than 90\% bootstrap probability.

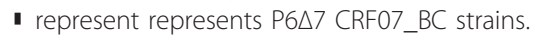

first time to establish the transmission linkage for P $6 \Delta 7$ strains; Importantly, the transmission has occurred in different epidemic regions. Therefore, these data serve as a wake-up call for our authority. Since the transmission of P6 $\Delta 7$ strains was only observed between epidemiologically closely related IDUs, it is speculated that this is the initial phase for the transmission of $\mathrm{P} 6 \Delta 7$ strains. In addition, our data also established that P6 67 CRF07_BC could be originated from different parental strains and thereby had versatile original ancestors in evolution. 
Both the independent occurrence of the P $6 \Delta 7$ in different CRF07_BC infected individuals and the transmission of P $6 \Delta 7$ strain among IDUs suggested that this deletion may have important implications. As known, HIV-1 Gag p6 protein play a critical role in viral particle budding by interaction with host factor Tsg101 and ALIX [15-18], there may exist active mechanisms for host cells to interrupt this process and thereby block the viral budding. Therefore, it is rationalized that P $6 \Delta 7$ may represent a new recombinant form escaping from anti-p6 based budding mechanism. In this regards, it will be important to address how P $6 \Delta 7$ will influence the engagement of $\mathrm{p} 6$ into the budding process.

\section{Additional material}

Additional file 1: Comparison of viral load and viral load change between non-deletion and P $6 \Delta 7$ CRF07_BC strains infected patients 11 non-deletion strains patients and 7 P6 67 CRF07_BC strains patients was consecutively follow-up for 2-3 years. No significance difference was detected in the initial viral load(infection time $<6$ months) of these two groups, whereas viral load of P6 7 was higher and increases more rapidly than that of non-deletion in last follow-up $(P<0.05)$.

\section{List of abbreviations}

HIV: Human Immunodeficiency Virus; CRF: Circulating recombinant form; IDU: Injection Drug User

\section{Acknowledgements}

This study was supported by NSFC Grant 30901257 and Chinese National Grand Program on Major new Drugs (Project number 2009ZX09303-006) and Key Infectious Disease Control and Prevention (2008ZX10001-002 and 015). It was also supported by China Postdoctoral Science Foundation (Grant No 201003233), Shanghai Postdoctoral Program (Grant no10R21410600).

\section{Author details}

'Shanghai Public Health Clinical Center, Institutes of Biomedical Sciences, Fudan University, 2901 Caolang Road, Research Center, Jinshan District, Shanghai, China, 201508. 'Zhejiang Provincial Key Laboratory of Medical Genetics, School of Laboratory Medicine of Wenzhou Medical College, University-town, Wenzhou, Zhejiang Province, China, 325035. ${ }^{3}$ Key Laboratory of Infectious Disease Prevention and Control, China CDC, Beijing, China.

\section{Authors' contributions}

MZ conceived the study, carried out the molecular genetic studies, participated in the sequence alignment and drafted the manuscript. $\mathrm{HH}$ and QC and SJ participated in the sequence alignment and participated in the design of the study and performed the probability testing of phylogenetic tree. $L J$ coordinated the study, participated in the experimental design and helped to draft of the manuscript. XJ and ZX proposed the concept of the study, designed the study, formulated the major conclusion and revised this manuscript, and all authors read and approved the final manuscript.

\section{Competing interests}

The authors declare that they have no competing interests.

Received: 13 November 2010 Accepted: 10 February 2011

Published: 10 February 2011

\section{References}

1. Coren LV, Thomas JA, Chertova E, Sowder RC, Gagliardi TD, Gorelick RJ, Ott DE: Mutational analysis of the C-terminal gag cleavage sites in human immunodeficiency virus type 1. J Virol 2007, 81:10047-10054.
2. Pikora CA, Wittish C, Desrosiers RC: p6 gag of human and simian immunodeficiency viruses is tolerant to small in-frame deletions downstream of the late domain. Virology 2006, 346:479-489.

3. Bleiber $G$, Peters S, Martinez R, Cmarko D, Meylan P, Telenti A: The central region of human immunodeficiency virus type 1 p6 protein (Gag residues S14-131) is dispensable for the virus in vitro. J Gen Virol 2004, 85:921-927.

4. Yan-Hui Song, ZheFeng Meng, Xing Hui, Yuhua Ruan, Xinping Li, Ruolei Xin, Pengfei Ma, Peng Hong, Shao Yiming: Analysis of HIV-1 CRF07_BC gag p6 sequences indicating novel deletions in the central region of p6. Arch Virol 2007, 26:1553-1558.

5. Wang L: Overview of the HIV/AIDS epidemic, scientific research and government responses in China. AIDS 2007, 21(Suppl 8):S3-7.

6. Zhang KL, Ma SJ: Epidemiology of HIV in China [letter]. BMJ 2002, 324:803-804.

7. Lin YT, Lan YC, Chen YJ: Molecular epidemiology of HIV-1 infection and fulllength genomic analysis of circulating recombinant form 07_BC strains from injection drug users in Taiwan. J Infect Dis 2007, 195:1283-1293.

8. Piyasirisilp S, McCutchan FE, Carr JK, Sanders-Buell E, Liu W, Chen J, Wagner R, Wolf $H$, Shao $Y$, Lai S, Beyrer C, Yu XF: A recent outbreak of human immunodeficiency virus type 1 infection in southern China was initiated by two highly homogeneous, geographically separated strains, circulating recombinant form $\mathrm{AE}$ and a novel $\mathrm{BC}$ recombinant. J Virol 2000, 74:11286-11295.

9. Jia M, Luo H, Ma Y, Wang N, Smith K, Mei J, Lu R, Lu J, Fu L, Zhang Q, Wu Z, Lu L: The HIV epidemic in Yunnan Province, China, 1989-2007. J Acquir Immune Defic Syndr 2010, 539(Suppl 1):S34-40.

10. Posada D, Buckley TR: Model selection and model averaging in phylogenetics: advantages of the AIC and Bayesian approaches over likelihood ratio tests. Systematic Biology 2004, 53:793-808.

11. Paraskevis D, Magiorkinis E, Magiorkinis G, Kiosses VG, Lemey P, Vandamme AM, Rambaut A, Hatzakis A: Phylogenetic reconstruction of a known HIV-1 CRF04_cpx transmission network using maximum likelihood and Bayesian methods. J Mol Evol 2004, 59:709-717.

12. Shimodaira H, Hasegawa M: CONSEL: for assessing the confidence of phylogenetic tree selection. Bioinformatics 2001, 17:1246-1247.

13. Resik Sonia, Lemey Philippe, Ping Li-hua, Kouri Vivian, Joanes Jose, Pérez Jorge, Vandamme AM, Swanstrom R: Limitations to Contact Tracing and Phylogenetic Analysis in Establishing HIV Type 1 Transmission Networks in Cuba. AIDS Res Hum Retroviruses 2007, 23:347-356.

14. Yang Z, Rananala B: Bayesian phylogenetic inference using DNA sequences: A Markov Chain Monte Carlo method. Mol Biol Evol 1997, 14:717-724.

15. Lazert C, Chazal N, Briant L, Gerlier D, Cortay JC: Refined study of the interaction between HIV-1 p6 late domain and ALIX. Retrovirology 2008, 13:39

16. Dussupt V, Javid MP, Abou-Jaoudé G, Jadwin JA, de La Cruz J, Nagashima K, Bouamr F: The nucleocapsid region of HIV-1 Gag cooperates with the PTAP and LYPXnL late domains to recruit the cellular machinery necessary for viral budding. PLoS Pathog 2009, 5:e1000339.

17. Strack B, Calistri A, Craig S, Popova E, Göttlinger HG: AIP1/ALIX is a binding partner for HIV-1 p6 and EIAV p9 functioning in virus budding. Cell 2003, 114:689-699.

18. Fisher RD, Chung HY, Zhai Q, Robinson H, Sundquist WI, Hill CP: Structural and biochemical studies of ALIX/AIP1 and its role in retrovirus budding. Cell 2007, 128:841-852.

doi:10.1186/1743-422X-8-60

Cite this article as: Zhefeng et al: Transmission of new CRF07 BC Strains with 7 amino acid deletion in Gag p6. Virology Journal 2011 8:60. 